\title{
OPTIMAL CONTROL OF STRUCTURE SUBJECT TO EARTHQUAKE LOADING USING DYNAMIC PROGRAMMING
}

\author{
By Mutsuto KAWAHARA* and Keiji FUKAZAWA**
}

\begin{abstract}
The purpose of this paper is to present an investigation of the optimal control of structure subject to earthquake loading. The control of structure in civil engineering results in the tracking problem, so that this paper deals with the solution of the tracking problem for the descrete-time linear quadratic control. The dynamic programming is used for the solution algorithm. Several numerical examples comparing the regulator control and various approximating control methods lead to the conclusion that the structure can be efficiently controlled by the methods presented in this paper.
\end{abstract}

Keywords: optimal control, earthquake loading, dynamic programming

\section{INTRODUCTION}

In recent years, a number of researches to control the response of structures subject to earthquake loading applying the artificial control force resisted to the external loading have been presented ${ }^{1)-4)}$. Almost all of the optimal controls presented previously are based on the regulator control. Namely, the control is carried out based on the differential equation of state which does not include the non-homogeneous term resulting from the earthquake load. Contrary to this, the optimal control of structure subject to earthquake loading should be treated as the tracking problem, which includes the non-homogeneous term in the equation of state derived from the earthquake loading ${ }^{5) .6)}$.

The aim of this paper is to present an investigation of the optimal control of structure by the tracking control based on the dynamic programming. In the optimal control of structure, the performance index to estimate the efficiency of control should be introduced. For this index, the quadratic integral value of structural response and control force is used. Because the structural response and control force can not be small simultaneously, control force is determined as to minimize the performance index so that the appropriate relation between structural response and control force can be found. For the solution algorithm, the dynamic programming is effectively used.

In the tracking control system, it is neccessary to know all of the external load in advance to determine the optimal control force. But, in case of earthquake, for example, it is almost impossible to obtain all of the external load in advance. Since the optimal control force can not be determined before or on occassion of the earthquake, this control system is inconvenient to use for an actual control. However, in other words it can be said that the optimal control force in the tracking control system is the most effective

* Member of JSCE, Dr. Eng., Professor, Dept. of Civil Engng., Chuo Univ., (Bunkyo-ku, Tokyo)

** Member of JSCE, Graduate Student Dept. of Civil Engng., Chuo Univ., (Bunkyo-ku, Tokyo) 
control force because it utilizes all of the external loads. Therefore, the computed results obtained by the tracking control are considered the standard results for comparative study with the results of other control systems. Thus, the results can be considered as one of the important information in the field of engineering. In this paper, the sub-tracking and instantaneous controls are also introduced for the purpose of actual controls. Those control systems are based on the solution algorithm of the tracking control obtained by the dynamic programming. The computed results obtained by the regulator control are also represented for comparison. By numerical examples carried out in this paper. It is concluded that the sub-tracking control knowing the earth quake load back to short time before the control instant results in almost the same as that obtained by the tracking control.

\section{CONTROL PROBLEM}

In this paper, the linear quadratic control technique with fixed terminal time is applied to the control of structure subject to an earthquake loading. The equation of motion of the controlled structure can be described as follows.

$$
[M]\{\ddot{y}\}+[C]\{\dot{y}\}+[K]\{y\}=[V]\{u\}-[M]\{E\} \ddot{\varphi}
$$

where $[M],[C]$ and $[K]$ denote mass, damping and stiffness matrices, respectively, $[V]$ is the matrix which gives number and place of controllers, $\{y\}$ and $\{u\}$ express displacement and control force vectors, and $\{E\}$ is the vector which gives the direction of an earthquake, and $\ddot{\varphi}$ is earthquake acceleration. The mass $[M]$ and the stiffness $[K]$ matrices can be derived by the conventional finite element method. For the damping matrix $[C]$, the appropriate assumption may be introduced in the later section. The performance index is defined as follows.

$$
J=\frac{1}{2} \int_{0}^{t_{f}}\left[\left\{\begin{array}{c}
y(t) \\
\dot{y}(t)
\end{array}\right\}^{T} S\left\{\begin{array}{c}
y(t) \\
\dot{y}(t)
\end{array}\right\}+\{u(t)\}^{T} T\{u(t)\}\right] d t
$$

where $t_{f}$ denotes the terminal time, and $S, T$ are the weighting matrices. The performance index $J$ is composed of quadratic integral values of the structural response and control force, which are contradictory each other. Namely, according as the control force tends larger, the structural response may be smaller. Both structural response and control force can not be small simultaneously. The optimal control of structure is to find the appropriate relation between structural response and control force. Therefore, the control problem in this paper is defined to find the vector $\{u\}$ which minimizes the value of $J$ in Eq. (2) under the condition of Eq. (1) with the appropriate boundary and initial conditions. Because Eqs. (1) and (2) are the control system, in which non-homogeneous term derived from the earthquake loading is included, this type of control system is called as the tracking system.

\section{MODAL ANALYSIS OF EQUATION OF MOTION}

Eq. ( 1 ) can be transformed into the discrete form by the modal analysis. The circular natural frequency $\omega$ can be determined by

$$
\omega^{2}[M]\{y\}=[K]\{y\}
$$

The modal matrix $[Y]$, that is the assemble of the colum matrix of the modal vector derived by Eq. (3), is obtained and can be normalized as follows.

$[Y]^{T}[M][Y]=[I], \quad[Y]^{T}[K][Y]=\left[\omega^{2}\right]$

where the matrix $[I]$ denotes the unit matrix, and expression [ ] means the diagonal matrix. Here, the transformation is introduced as follows.

$$
\{y\}=[Y]\{q\}, \quad\{u\}=[V]^{T}[Y]\{x\}
$$

where $\{q\}$, and $\{x\}$ represent the modal displacement and modal control force vector respectively. Substituting Eqs. (6) and (7) into Eq. (1), premultiplying by [Y], and assumming that the damping matrix $[C]$ is

$$
[Y]^{T}[C][Y]=2 h[\omega]
$$


in which $h$ is the damping constant, the decoupled equation of motion can be described in the following form

$$
\{\ddot{q}\}+2 h[\omega]\{\dot{q}\}+\left[\omega^{2}\right]\{q\}=[Y]^{T}[V][V]^{T}[Y]\{x\}-[Y]^{T}[M]\{E\} \ddot{\varphi}
$$

Let the total time $\left[0, t_{f}\right]$ to be analized be divided into a number of short time intervals $\Delta t$ by time points, one of which is expressed by $k$. In the short time interval $\Delta t$, the earthquake acceleration may be assumed as a linear function of time $t$ :

$$
\ddot{\varphi}=a t+b
$$

where $a$ and $b$ are given constants. Substituting Eq. (10) into Eq. (9), and integrating Eq. (9), the modal coordinate and modal velocity at the $k+1$ th time stage $q(k+1), \dot{q}(k+1)$ are given in the following form.

$$
\begin{aligned}
& {\left[\begin{array}{l}
\{q(k+1)\} \\
\{\dot{q}(k+1)\}
\end{array}\right]=\left[\begin{array}{cc}
F_{x}^{\prime} & G_{x}^{\prime} \\
F_{v}^{\prime} & G_{v}^{\prime}
\end{array}\right]\left[\begin{array}{l}
\{q(k)\} \\
\{\dot{q}(k)\}
\end{array}\right]+\left[\begin{array}{c}
E_{x}^{\prime} \\
E_{v}^{\prime}
\end{array}\right][Y]^{T}[V][V]^{T}[Y]\{x(k)\}-\left[\left[\begin{array}{c}
D_{x}^{\prime} \\
D_{v}^{\prime}
\end{array}\right][Y]^{T}[M]\{E\} a_{k}\right.} \\
& \left.+\left[\begin{array}{c}
E_{x}^{\prime} \\
E_{v}^{\prime}
\end{array}\right][Y]^{T}[M]\{E\} b_{k}\right]
\end{aligned}
$$

where

$$
\begin{aligned}
& F_{x}^{\prime}=\frac{1}{\omega \sqrt{1-h^{2}}} \exp (-h \omega t) \cos \left(\omega \sqrt{1-h^{2}} t-\varphi_{F}\right) \\
& F_{v}^{\prime}=-\frac{\omega}{\sqrt{1-h^{2}}} \exp (-h \omega t) \cos \left(\omega \sqrt{1-h^{2}} t-\left(\varphi_{F}+\varphi\right)\right) \\
& G_{x}^{\prime}=\frac{1}{\omega \sqrt{1-h^{2}}} \exp (-h \omega t) \sin \left(\omega \sqrt{1-h^{2}} t\right) \\
& G_{v}^{\prime}=-\frac{1}{\sqrt{1-h^{2}}} \exp (-h \omega t) \sin \left(\omega \sqrt{1-h^{2}} t-\varphi\right) \\
& E_{x}^{\prime}=-\frac{1}{\omega^{2} \sqrt{1-h^{2}}} \exp (-h \omega t) \cos \left(\omega \sqrt{1-h^{2}} t-\varphi_{E}\right)+\frac{1}{\omega^{2}} \\
& E_{v}^{\prime}=\frac{1}{\omega \sqrt{1-h^{2}}} \exp (-h \omega t) \cos \left(\omega \sqrt{1-h^{2}} t-\left(\varphi_{E}+\varphi\right)\right) \\
& D_{x}^{\prime}=\frac{1}{\omega^{3} \sqrt{1-h^{2}}} \exp (-h \omega t) \cos \left(\omega \sqrt{1-h^{2}} t-\varphi_{D}\right)+\frac{t}{\omega^{2}}-\frac{2 h}{\omega^{3}} \\
& D_{v}^{\prime}=\frac{1}{\omega^{2} \sqrt{1-h^{2}}} \exp (-h \omega t) \cos \left(\omega \sqrt{1-h^{2}} t-\left(\varphi_{D}+\varphi\right)\right)+\frac{1}{\omega^{2}} \\
& \varphi_{D}=\tan ^{-1} \frac{2 h^{2}-1}{2 h \sqrt{1-h^{2}}} \quad \varphi_{E}=\varphi_{F}=\tan ^{-1} \frac{h}{\sqrt{1-h^{2}}} \quad \varphi^{-1} \frac{\sqrt{1-h^{2}}}{h}
\end{aligned}
$$

in which $q(k)$ and $\dot{q}(k)$ denote the modal coordinate and the modal velocity at the $k$ th time stage respectively.

\section{OPTIMAL CONTROL OF STRUCTURE IN DISCRETE TIME}

Regarding the calculated displacement and velocity vectors at the $k$ th time stage as the initial vectors of the $k+1$ th time stage, the displacement and velocity vectors at the $k+1$ th time stage can be rewritten in the following equation.

$$
X(k+1)=A X(k)+B U(k)+C(k), \quad X(0)=\left[\begin{array}{l}
\{q(0)\} \\
\{\dot{q}(0)\}
\end{array}\right]
$$

where

$$
k=0,1,2, \cdots, N-3, N-2, N-1
$$

$$
A=\left[\begin{array}{cc}
F_{x}^{\prime} & G_{x}^{\prime} \\
F_{v}^{\prime} & G_{v}^{\prime}
\end{array}\right] \quad C(k)=-\left[\left[\begin{array}{c}
D_{x}^{\prime} \\
D_{v}^{\prime}
\end{array}\right][Y]^{T}[M]\{E\} a_{k}+\left[\begin{array}{c}
E_{x}^{\prime} \\
E_{v}^{\prime}
\end{array}\right][Y]^{T}[M]\{E\} b_{k}\right]
$$




$$
\begin{aligned}
& B=\left[\begin{array}{c}
E_{x}^{\prime} \\
E_{v}^{\prime}
\end{array}\right][Y]^{T}[V][V]^{T}[Y] \quad X(k)=\left[\begin{array}{l}
\{q(k)\} \\
\{\dot{q}(k)\}
\end{array}\right] \\
& U(k)=\{x(k)\}
\end{aligned}
$$

Eq. (12) is generally referred to as the discrete-time equation of state, and defined from 0 th to $N-1$ th time stages. This equation is composed of the modal coordinate, modal velocity and modal control force. The discrete-time performance index, which is a function of the modal coordinate, modal velocity and modal control force, can be written as follows.

$$
J=\sum_{k=0}^{N-1}\left\{X^{T}(k+1) Q X(k+1)+U^{T}(k) R U(k)\right\}
$$

where

$$
Q=Y^{T} S Y, \quad R=V^{T} Y^{T} T Y V
$$

The optimal control of structure is redefined to find out the generalized optimal control force which minimizes the performance index $J$ of Eq. (13) under the constraint of Eq. (12). The generalized optimal control force is specified in each vibration mode. The final optimal control force is determined by superposing the generalized optimal control force in each vibration mode. If the optimal control force can be obtained in the vibration mode, the modal coordinate and the modal velocity can be calculated by Eq. (12). The original displacement and velocity are determined by superposing the modal coordinate and the modal velocity respectively.

\section{DYNAMIC PROGRAMMING}

To determine the optimal force, the dynamic programming techique has been employed ${ }^{7}$. Total time of the optimal control problem is divided into $N$ time stage, i. e., from 0 to $N-1$. The performance index of $k$ th time stage $j$ is defined as follows.

$$
j_{k}=\left[X^{T}(k+1) Q X(k+1)+U^{T}(k) R U(k)\right]
$$

and, also $J_{k}$ is defined in the following form.

$$
J_{k}=j_{k}+j_{k+1}+\cdots+j_{N-1}
$$

If $k$ is put zero in Eq. (15), then $J_{k}$ is completely coincident with $J$ in Eq. (13). At $N-1$ th time stage, the optimal control force $U_{O P T}(N-1)$ is determined as to minimize $J_{N-1}$. Then at $N-2$ th time stage the optimal control force $U_{O P T}(N-2)$ is also chosen as to minimize the index $j_{N-1}+\min J_{N-1}$. This operation can be carried out until 0th time stage, according to the routine of the dynamic programming shown in Fig. 1. As a result, all of the optimal control forces can be obtained. In each time stage optimal control force can be derived in the following way.

(1) $k=N-1$

The performance index at $N-1$ th time stage can be written as:

$$
J_{N-1}=\left[X^{T}(N) Q X(N)+U^{T}(N-1) R U(N-1)\right]
$$

Using Eq. (15), one obtains that

$$
J_{N-1}=j_{N-1}
$$

The optimal control force $U_{O P T}(N-1)$ is determined as to minimize $J_{N-1}$. The optimal control force $U_{O P T}(N-1)$ can be written as ;

$$
U_{o P T}(N-1)=-\left(R+B^{T} Q B\right) B^{T}\{Q A X(N-1)+Q C(N-1)\}
$$

Substituting Eq. (17) into Eq. (16), $\min J_{N-1}$ can be obtained.

$$
\min J_{N-1}=X^{T}(N-1) w_{1} X(N-1)+X^{T}(N-1) Y_{1} C(N-1)
$$

$$
+C^{T}(N-1) Y_{1}^{T} X(N-1)+C^{T}(N-1) Z_{1} C(N-1)
$$

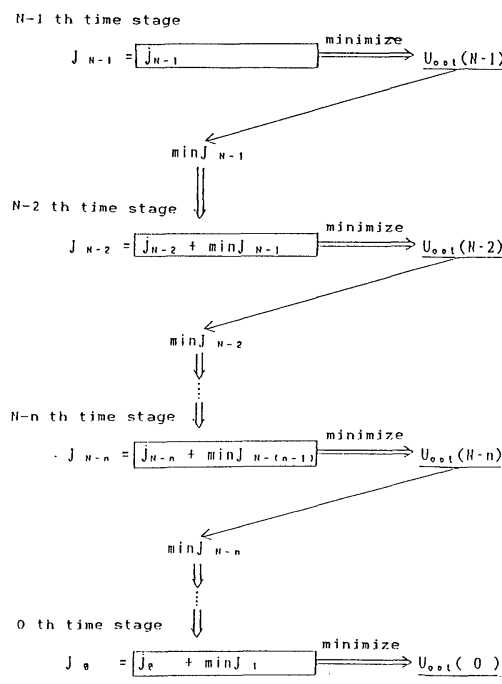

Fig. 1 Routine of Dynamic Programming. 
where

$$
\begin{aligned}
& w_{1}=A^{T} Q A-A^{T} Q B\left(R+B^{T} Q B\right)^{-1} B^{T} Q A \\
& Y_{1}=A^{T} Q-A^{T} Q B\left(R+B^{T} Q B\right)^{-1} B^{T} Q \\
& Z_{1}=Q-Q B\left(R+B^{T} Q B\right)^{-1} B^{T} Q
\end{aligned}
$$

After the transformation $W_{0}=Q$ and $W_{1}=w_{1}+Q W_{1}, \quad Y_{1}, Z_{1}$ become:

$$
\begin{aligned}
& W_{1}=Q+A^{T} W_{0} A-A^{T} W_{0} B\left(R+B^{T} W_{0} B\right)^{-1} B^{T} W_{0} A \\
& Y_{1}=A^{T} W_{0}-A^{T} W_{0} B\left(R+B^{T} W_{0} B\right)^{-1} B^{T} W_{0} \\
& Z_{1}=W_{0}-W_{0} B\left(R+B^{T} W_{0} B\right)^{-1} B^{T} W_{0}
\end{aligned}
$$

(2) $k=N-2$

The performance index at $N-2$ th time stage can be written as

$$
J_{N-2}=\left[X^{T}(N-1) Q X(N-1)+U^{T}(N-2) R U(N-2)\right]
$$

Using Eq. (15), one obtains that

$$
J_{N-2}=j_{N-2}+j_{N-1}
$$

By the principle of optimality the optimal control force $U_{O P T}(N-2)$ can be determined as to mini-mize $J_{N-2}$ :

$$
J_{N-2}=\left[j_{N-2}+\min J_{N-1}\right]
$$

The optimal control force $U_{O P T}(N-2)$ can be expressed as :

$$
U_{\text {oPT }}(N-2)=-\left(R+B^{T} W_{1} B\right)^{-1} B^{T}\left\{W_{1} A X(N-2)+W_{1} C(N-2)+Y_{1} C(N-1)\right\}
$$

Substituting Eq. (19) into Eq. (18), $\min J_{N-2}$ can be obtaind.

$$
\begin{aligned}
\min J_{N-2}= & {\left[X^{T}(N-2) W_{2} X(N-2)+X^{T}(N-2) Y_{2} C(N-2)+C^{T}(N-2) Y_{2}^{T} X(N-2)\right.} \\
& +C^{T}(N-2) Z_{2} C(N-2)+X^{T}(N-2) \alpha_{2} C(N-1)+C^{T}(N-1) \alpha_{2}^{T} C(N-2) \\
& \left.+C^{T}(N-2) \beta_{2} C(N-1)+C^{T}(N-1) \beta_{2}^{T} C(N-2)+C^{T}(N-1) \gamma_{2} C(N-1)\right]
\end{aligned}
$$

where

$$
\begin{array}{ll}
W_{2}=Q+A^{T} W_{1} A-A^{T} W_{1} B\left(R+B^{T} W_{1} B\right)^{-1} B^{T} W_{1} A & \alpha_{2}=A^{T} Y_{1}-A^{T} W_{1} B\left(R+B^{T} W_{1} B\right)^{-1} B^{T} Y_{1} \\
Y_{2}=A^{T} W_{1}-A^{T} W_{1} B\left(R+B^{T} W_{1} B\right)^{-1} B^{T} W_{1} & \beta_{2}=Y_{1}-W_{1} B\left(R+B^{T} W_{1} B\right)^{-1} B^{T} Y_{1} \\
Z_{2}=W_{1}-W_{1} B\left(R+B^{T} W_{1} B\right)^{-1} B^{T} W_{1} & \gamma_{2}=Z_{1}-Y_{1} B\left(R+B^{T} W_{1} B\right)^{-1} B^{T} Y_{1}
\end{array}
$$

in which $Y_{1}$ is as follows:

$$
Y_{1}=A^{T} W_{0}-A^{T} W_{0} B\left(R+B^{T} W_{0} B\right)^{-1} B^{T} W_{0}
$$

$U_{O P T}(N-2)$ can be transformed into the following form.

$$
\begin{aligned}
U_{O P T}(N-2)= & -\left(R+B^{T} W_{1} B\right)^{-1} B^{T}\left[W_{1} A X(N-2)+W_{1} C(N-2)\right. \\
& \left.+\left\{A^{T}-A^{T} W_{0} B\left(R+B^{T} W_{0} B\right)^{-1} B^{T}\right\} W_{0} C(N-1)\right]
\end{aligned}
$$

(3) $k=N-3$

The optimal control force $U_{O P T}(N-3)$ can be obtaind as:

$$
\begin{aligned}
U_{O P T}(N-3)= & -\left(R+B^{T} W_{2} B\right)^{-1} B^{T}\left[W_{2} A X(N-3)+W_{2} C(N-3)\right. \\
& +\left\{A^{T}-A^{T} W_{1} B\left(R+B^{T} W_{1} B\right)^{-1} B^{T}\right\} W_{1} C(N-2)+\left\{A^{T}-A^{T} W_{1} B\left(R+B^{T} W_{1} B\right)^{-1} B^{T}\right\} \\
& \left.\times\left\{A^{T}-A^{T} W_{0} B\left(R+B^{T} W_{0} B\right)^{-1} B^{T}\right\} W_{0} C(N-1)\right]
\end{aligned}
$$

(4) $k=N-n$

Similary, the general term of optimal control force at $N-n$th time stage can be expressed in the following form.

$$
\begin{aligned}
U_{O P T}(N-n)=-\left(R+B^{T} W_{n-1} B\right)^{-1} & B^{T}\left[W_{n-1} A X(N-n)\right. \\
& +W_{n-1} C(N-n) \\
& +Y_{n-2} W_{n-2} C\{(N-(n-1))\} \\
& +Y_{n-2} Y_{n-3} W_{n-3} C\{(N-(n-2))\} \\
& +Y_{n-2} Y_{n-3} Y_{n-4} W_{n-4} C\{(N-(n-3))\} \\
& +Y_{n-2} Y_{n-3} Y_{n-4} Y_{n-5} W_{n-5} C\{(N-(n-4))\} \\
& \vdots \\
& \left.+Y_{n-2} Y_{n-3} Y_{n-4} Y_{n-5} Y_{n-6} \cdots Y_{2} Y_{1} Y_{0} W_{0} C(N-1)\right]
\end{aligned}
$$




$$
\begin{aligned}
& W_{0}=Q \\
& W_{n}=Q+A^{T} W_{n-1} A-A^{T} W_{n-1} B\left(R+B^{T} W_{n-1} B\right)^{-1} B^{T} W_{n-1} A \\
& Y_{n}=A^{T}-A^{T} W_{n} B\left(R+B^{T} W_{n} B\right)^{-1} B^{T} \ldots \ldots \ldots \ldots \ldots \ldots \ldots \ldots \ldots \ldots \ldots \ldots \ldots \ldots \ldots \ldots \ldots
\end{aligned}
$$

where $\mathrm{Eq} .(21)$ is generally referrred to as the discrete-time Ricatti equation.

\section{SUB-TRACKING AND INSTANTANEOUS OPTIMAL CONTROLS OF STRUCTURE}

Using equation (20), the control force at the first time stage can be expressed as follows.

$$
\begin{aligned}
U_{\text {oPT }}(0)=-\left(R+B^{T} W_{N-1} B\right)^{-1} & B^{T}\left\{W_{N-1} A X(0)\right. \\
& +W_{N-1} C(0) \\
& +Y_{N-2} W_{N-2} C(1) \\
& +Y_{N-2} Y_{N-3} W_{N-3} C(2) \\
& +Y_{N-2} Y_{N-3} Y_{N-4} W_{N-4} C(3) \\
& +Y_{N-2} Y_{N-3} Y_{N-4} Y_{N-5} W_{N-5} C(4) \\
& \vdots \\
& \left.+Y_{N-2} Y_{N-3} Y_{N-4} Y_{N-5} Y_{N-6} \cdots Y_{2} Y_{1} Y_{0} W_{0} C(N-1)\right\}
\end{aligned}
$$

As is detected out in Eq. (23), it is obvious that the earthquake load during all control time duration must be known to determine the optimal control force at the first time stage $U_{O P T}(0)$. Contrary to this, it is almost impossible in the actual control problem of structure to control the structure on the condition that the earthquake load of all control time duration must be known at the beginning of the control. Therefore, it is strictly necessary to present the method in which the control of structure can be carried out knowing that a part of earthquake load or an instantaneous earthquake load, at the time only on which the control force will be applied. The control method in which only a part of earthquake load is required to be known in advance is referred to as the sub-tracking control system in this paper. The optimal control force of the sub-tracking method is derived from Eq. (23), neglecting the terms after the $i$ th time stage, in which $i$ means the time stage until which the earthquake load should be known. The optimal control force of the sub-tracking method can be written in the following form.

$$
\begin{aligned}
U_{O P T}(0)=-\left(R+B^{T} W_{N-1} B\right)^{-1} & B^{T}\left\{W_{N-1} A X(0)\right. \\
& +W_{N-1} C(0) \\
& +Y_{N-2} W_{N-2} C(1) \\
& +Y_{N-2} Y_{N-3} W_{N-3} C(2) \\
& +\vdots \\
& \left.+Y_{N-2} Y_{N-3} Y_{N-4} Y_{N-5} \cdots Y_{N-(i+1)} W_{N-(i+1)} C(i)\right\}
\end{aligned}
$$

where $W$ and $Y$ are the matrices depending on the number of control stages $N$. In case of the optimal control of structure of earthquake loading, it is almost impossible to know the number of control stages in advance. In the same reason, $W$ and $Y$ can not be obtained in advance. However, according as the number of control stages increase, $W$ and $Y$ can be considerd as constant matrices. Finally, the control force of the sub-tracking control at $k$ th time stage can be expressed in the following form.

$$
\begin{aligned}
& U_{O P T}(k)=-\left(R+B^{T} W_{S T} B\right)^{-1} B^{T}\left\{W_{S T} A X(k)+W_{S T} C(k)\right. \\
& +Y_{S T} W_{S r} C(k+1) \\
& +Y_{S T}^{2} W_{S T} C(k+2) \\
& \text { : } \\
& \left.+Y_{S T}^{i} W_{S T} C(k+i)\right\} \\
& k=0,1,2, \cdots, N-3, N-2, N-1
\end{aligned}
$$

where $W_{S T}$ means the constant matrix, which is the last value of the backword computation. The optimal control based on Eq. (25) can be carried out knowing the earthquake load until $i$ th time stage in advance. If the earthqeuake sensor is located at the place far from the structure, it is practically possible that the earthquake load before the $i$ th time stage will be known. If $i$ is put zero in Eq. (25), the instantaneous 
optimal control is obtained. Namely, the control force can be approximately determined using the measured earthquake load at the first time stage only, i. e.,

$$
\begin{array}{r}
U_{O P T}(k)=-\left(R+B^{T} W_{S T} B\right)^{-1} B^{T}\left\{W_{S T} A X(k)+W_{S T} C(k)\right\} \\
k=0,1,2, \cdots, N-3, N-2, N-1
\end{array}
$$

\section{FRAMED STRUCTURE USED IN THE NUMERICAL CALCULATION}

The ten story framed structure, as shown in Fig. 2, is used in the following numerical calculations. The column and beam characteristics of the structure are shown in Table 1. The consistent mass is used. The control force is applied on the roof. The natural frequencies and the vibration modes of this structure are shown in Fig. 3. The superposition is carried out for the first four modes. The earthquake subjecting to this structure is the El-Centro earthquake which is shown in Fig. 4. The maximum earthquake acceleration of this earthquake is 319 (gal).

\section{TRACKING CONTROL}

The numerical computations of control of structure by the tracking control system has been performed. The response of structural displacement and the control force are calculated assuming the following conditions. The weighting matrix $S$ that is related with the response quantity in the performance index is selected as a unit matrix. The values of the weighting matrix $T$ which affects the value of control force in the performance index is selected as $0.1,0.01$ and 0.001 .

The time histories of the controlled displacement of 5 th, 7 th, and 10 th floors of the structure and corresponding control forces varing $T=0.1$, and 0.001 are computed and illustrated inFigs. 5 and 6 . Solid line represents the controlled displacement. For comparison, uncontroled displacement conputed is shown in the figures by dotted line. The control force applied to the structure is also shown in the figures. Comparing these two figures, it is seen that the displacement of structure can be remarkably reduced according as the control force increases. For example, the maximum displacement of the top floor can be $97 \%$ reduced in case of $T=0.001$ comparing with that of the uncontrolled structure. Table 2 represents the maximum displacement at each floor and the maximum control force computed varing $T=0.1,0.01$ and 0.001 . The maximum displacement can be $6 \%$ reduced according as the maximum in an average control

Table 1 Column and beam characteristics of the structure.

\begin{tabular}{|c|c|c|c|c|}
\hline & \multicolumn{2}{|c|}{$\mathrm{col} \mathrm{umn}$} & \multicolumn{2}{|c|}{$\mathrm{b}$ e $\mathrm{am}$} \\
\hline & $A\left(\mathrm{~cm}^{2}\right)$ & $\mathrm{I}\left(\mathrm{C \textrm {m } ^ { 4 }}\right)$ & $A\left(\mathrm{~cm}^{2}\right)$ & $I\left(\mathrm{~cm}^{4}\right)$ \\
\hline $\begin{array}{l}1 F-4 F \\
5 F-7 F \\
8 F-10 F\end{array}$ & $\begin{array}{l}150.0 \\
120.0 \\
100.0\end{array}$ & $\begin{array}{l}3.125 \times 10^{4} \\
1.600 \times 10^{4} \\
0.333 \times 10^{4}\end{array}$ & $\begin{array}{l}100.0 \\
100.0 \\
100.0\end{array}$ & $\begin{array}{l}0.833 \times 10^{3} \\
0.833 \times 10^{3} \\
0.833 \times 10^{3}\end{array}$ \\
\hline \multicolumn{5}{|c|}{$\begin{aligned} \text { damping constant }= & 0.05 \\
\text { Young's modulous }= & 2.1 \times 10^{6}\left(\mathrm{~kg} / \mathrm{cm}^{2}\right) \\
& {\left[2.058 \times 10^{7}\left(\mathrm{~N} / \mathrm{cm}^{2}\right)\right] } \\
\text { unit weight per volume }=7.85 \times 10^{-3}\left(\mathrm{~kg} / \mathrm{cm}^{3}\right) & {\left[7.693 \times 10^{-2}\left(\mathrm{~N} / \mathrm{cm}^{3}\right)\right] }\end{aligned}$} \\
\hline
\end{tabular}
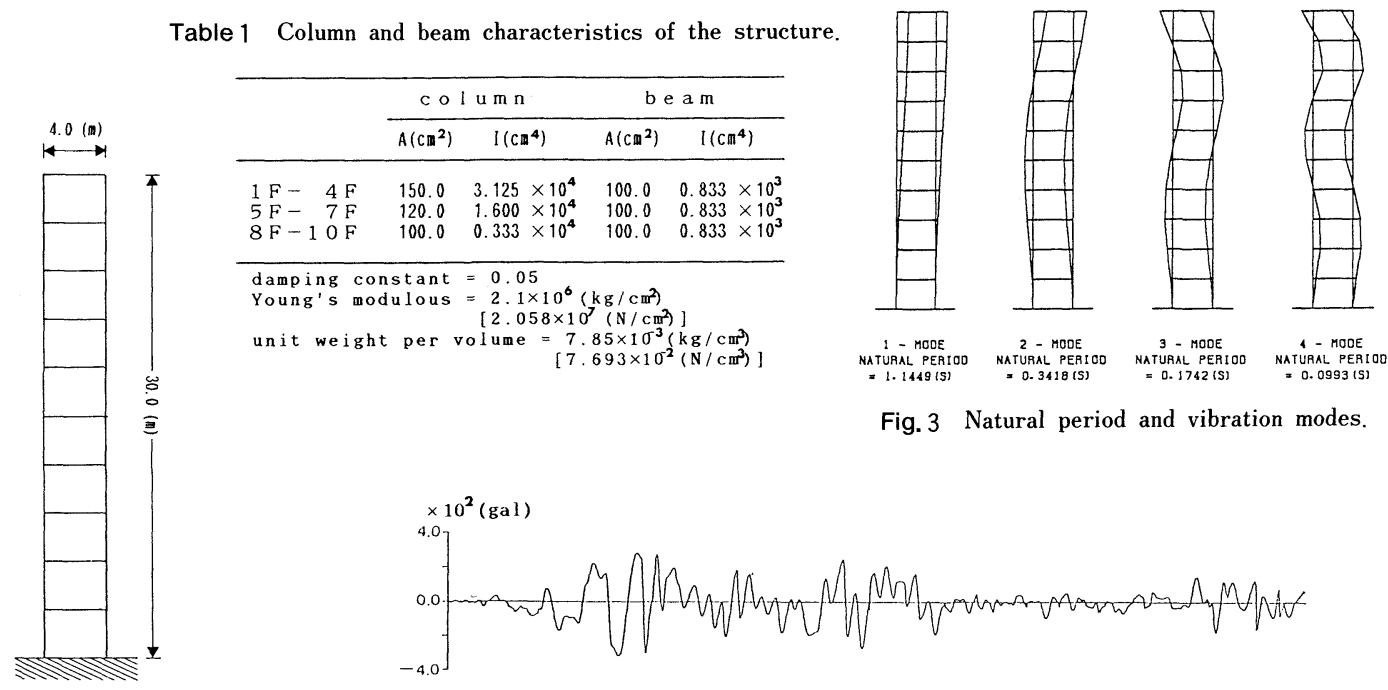

Fig. 3 Natural period and vibration modes.

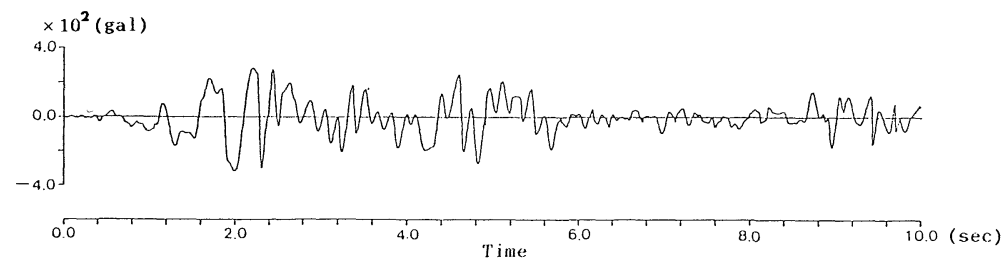

Fig. 2 The ten story framed structure.

Fig. 4 El-Centro Earthquake Accelation used in the tracking control. 

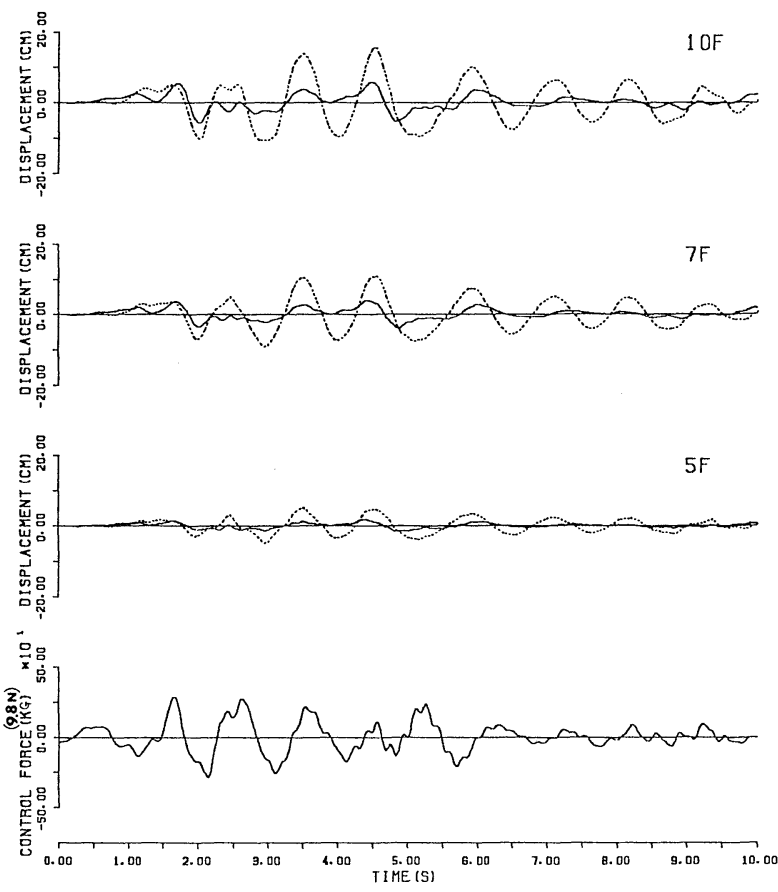

Fig. 5 Displacement and control force in the tracking control system $;=0.1$.
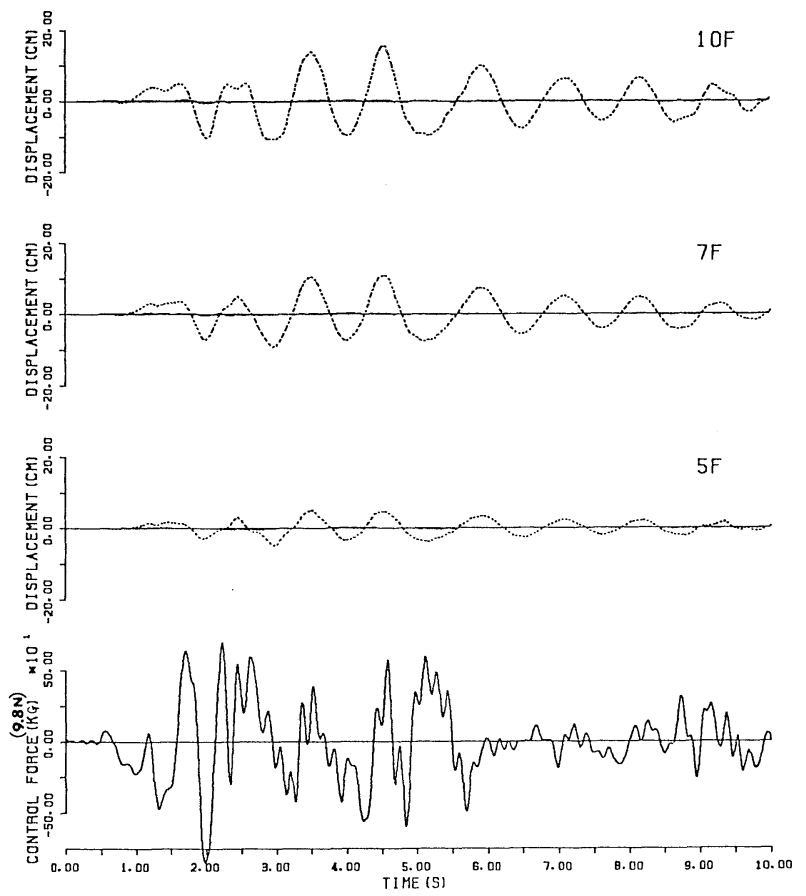

Fig. 6 Displacement and control force in the tracking control system ; $T=0.001$. 
Table 2 Maximum displacement of structure and maximum control force in the tracking control system.

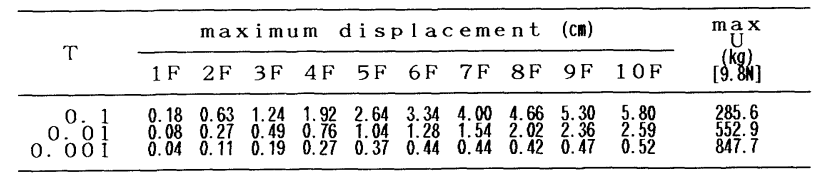

force $100(\mathrm{~kg})$ increases. In these numerical examples, it is found that there exists an appropriate control force such that the structural response can be reduced within the limited range.

\section{COMPARATIVE STUDY}

Comparison among the tracking control, sub-tracking control, instantaneous control and regulator control systems are carried out. The regulator control system is the closed-loop control so that the control force is regulated knowing only of the response of structure. Therefore, the control force of the regulator method is derived by Eq. (26) neglecting the terms of earthquake acceleration. This control system is often used in the conventional control analysis. For comparison, the results obtained by this method will be shown. In the sub-tracking control system, it is assumed that the earthquake load must be known in adavance until $i$ th time stage, where $i$ is a pre-assigned constant, which corresponds to the time $\Delta t \cdot i$ in an actual time. In the numerical computation presented in this paper, the time $\Delta t \cdot i$ is assumed as 0.2 . If the value of $i$ is taken to be zero, the instantaneous control system can be obrained. Namely, the instantaneous control system is the tracking system assumming that the non-homogeneous term dose not affect the response except the value at the time that the control force is applied.

It is necessary to introduce an index for comparative studies to evaluate the effectiveness of the control system. For this purpose, the quadratic integral value $J_{r}$ is introduced.

$$
J_{r}=\sum_{k=0}^{N-1}\left\{X^{T}(k+1) Q X(k+1)\right\}
$$

Namely, $J_{r}$ is the quadratic integral value of the structural response only. This value is not a function of control force. If $T$ varies, then the structural response obtained by each system varies. Therefore, $T$ can be chosen in the manner that the quadratic integral value $J_{r}$ is almost coincident with that of the tracking control system with $T=0.1$.

The time histories of the displacement at floors 5,7 and 10 of the structure and control force in each control system are illustrated in Figs. 7 9. Comparing the displacements and control forces in Fig. 5 with those in Fig. 7, it is seen that the displacements are almost coincident whereas the control forces are slightly different. Thus, the regulator control system is inefficient than the tracking control system. Comparing the results in Fig. 7 and those in Fig. 8, almost the same characterstics can be found. Comparing the results in Fig. 5 and those in Fig. 9, the structural displacement and control force in the sub-tracking control system are almost the same as those by the tracking control system.

The maximum displacement of the structure, the maximum control force, parameter $T$ and the quadratic integral value are shown in Table 3. Looking at the maximum control forces by the regulator and the instantaneous controls, the maximum value by the instantaneous control is $8.5 \%$ lower than that by the regulator control. Thus, the instantaneous control is more efficient than the regulator control. Comparing the resulted maximum structural displacements obtained by the tracking and the sub-tracking controls, almost the same value can be computed by both methods in spite that the maximum control forces are $7.8 \%$ different. Fig. 10 shows the diffrence of the control force between the tracking control and other control systems. In this figure, the discrepancy of the control system by the sub-tracking control is $71 \%$ smaller than that by the regulator control. Considering the facts stated in sections 8 and 9 , it is found that the control force by the sub-tracking control knowing the earthquake load back to 20th time stage before the 

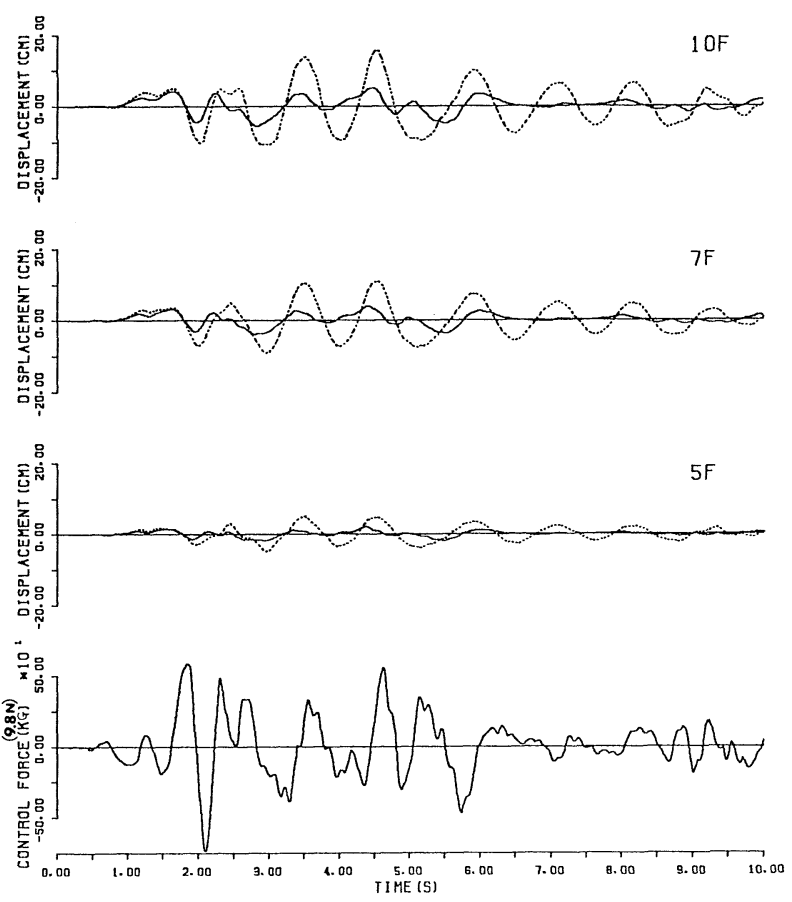

Fig. 7 Displacement and control force in the regulator control system.
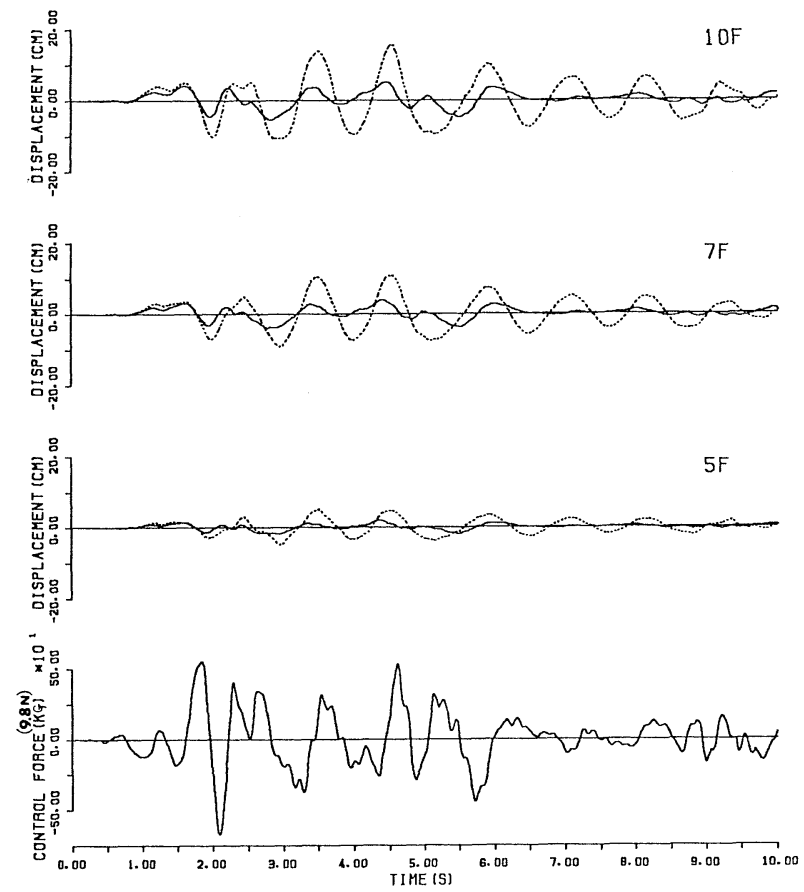

Fig. 8 Displacement and control force in the instnataneous control system. 

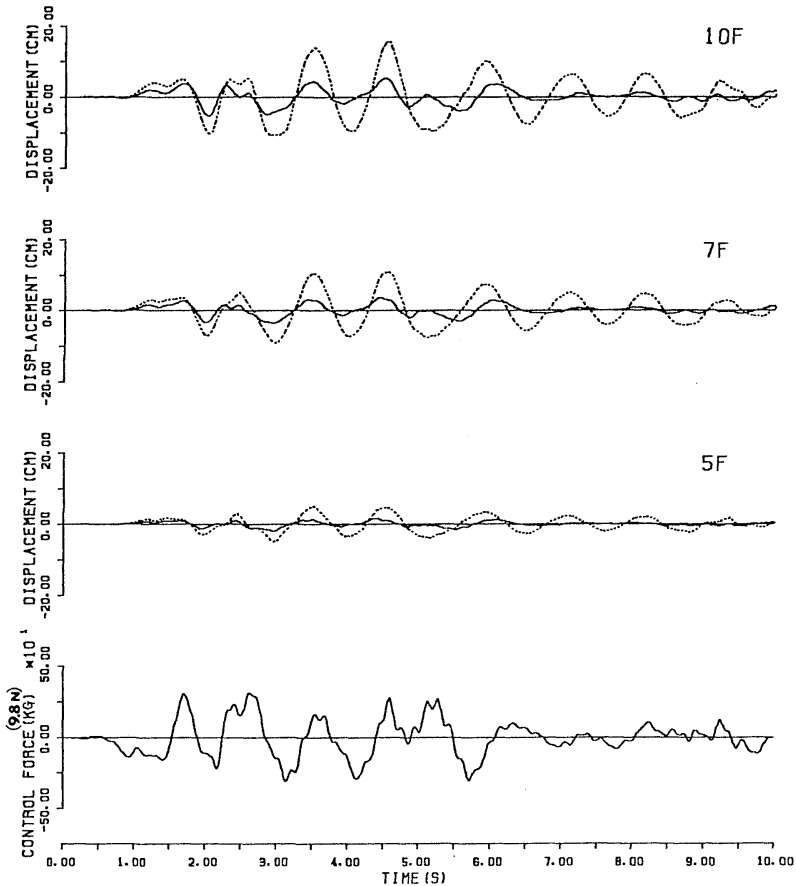

Fig. 9 Displacement and control force in the sub-tracking control system.

Table 3 Maximum displacement, control force and values $T$ and $J_{r}$ in eachcontrol system.

\begin{tabular}{|c|c|c|c|c|c|c|c|c|c|c|}
\hline & \multicolumn{2}{|c|}{ maximam } & \multicolumn{5}{|c|}{ displacement (cm) } & \multirow{2}{*}{$\underset{\left[\begin{array}{l}\max \\
{[9.8 \mathrm{~N}}\end{array}\right]}{\max }$} & \multirow{2}{*}{$\mathrm{T}$} & \multirow{2}{*}{$\mathrm{J}_{\mathrm{r}}$} \\
\hline & $1 \mathrm{~F}$ & $2 \mathrm{~F}$ & $3 \mathrm{~F}$ & $4 \mathrm{~F}$ & $5 \mathrm{~F}$ & $7 \mathrm{~F}$ & $10 \mathrm{~F}$ & & & \\
\hline $\begin{array}{l}\text { tracking control } \\
\text { regulator control } \\
\text { instantaneous control } \\
\text { sub-tracking control }\end{array}$ & $\begin{array}{l}0.18 \\
0.21 \\
0.21 \\
0.18\end{array}$ & $\begin{array}{l}0.63 \\
0.80 \\
0.70 \\
0.62\end{array}$ & $\begin{array}{l}1.24 \\
1.36 \\
1.35 \\
1.19\end{array}$ & $\begin{array}{l}1.92 \\
2.07 \\
2.05 \\
1.82\end{array}$ & $\begin{array}{l}2.64 \\
2.78 \\
2.75 \\
2.45\end{array}$ & $\begin{array}{l}4.00 \\
4.02 \\
4.00 \\
3.61\end{array}$ & $\begin{array}{l}5.80 \\
5.47 \\
5.50 \\
5.34\end{array}$ & $\begin{array}{l}285.6 \\
735.6 \\
673.2 \\
310.0\end{array}$ & $\begin{array}{l}0.100 \\
0.030 \\
0.036 \\
0.085\end{array}$ & $\begin{array}{l}743372.9 \\
743066.5 \\
744247.1 \\
743888.9\end{array}$ \\
\hline
\end{tabular}

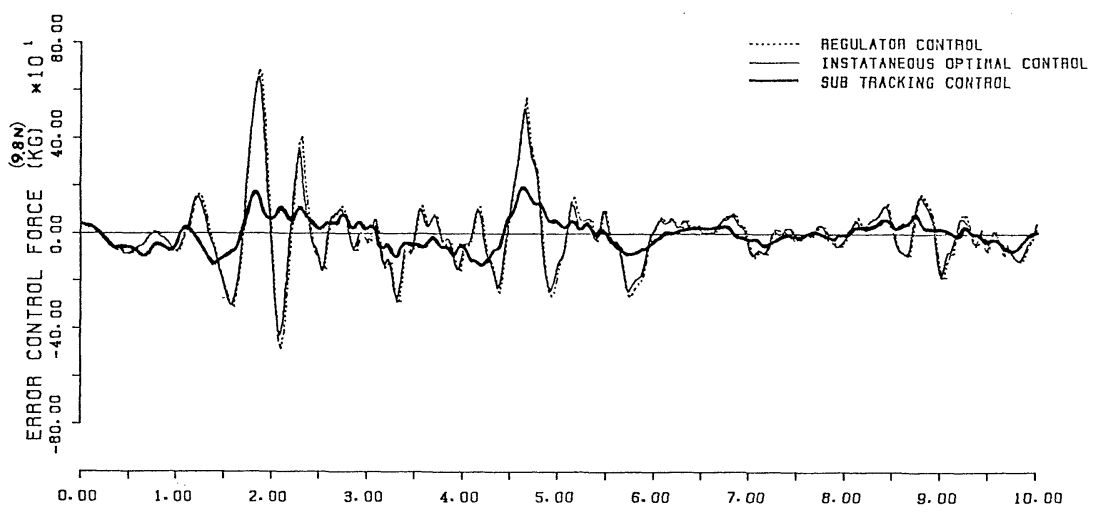

Fig. 10 Difference of control force between the tracking control and other control systems.

control instant is almost the same as that obtained by the tracking control.

\section{CONCLUSIONS}

The solution algorithm for the tracking problem of control of structure has been presented based on the 
dynamic programming. The outcomes derived by the numerical computations are as follows.

a) The displacement of structure can be remarkably reduced using the tracking control.

b) There exists an appropriate control force such that the structural response can be reduced within the limited range.

c) Using the sub-stracking control, almost the same control as the tracking control can be performed.

d) Even by the instantaneous control, the smaller control force can be obtained than that computed by the regulator control.

e) Regulator control is not recommendable because the control force obtained is $157 \%$ larger than that by the tracking control.

Considering the above outcomes, it is shown that the tracking control of structure can be effectively performed by the dynamic programming.

\section{REFERENCES}

1) Yang, J-N. : Application of Optimal Control Theory to Civil Engineering Structures, Journal of the Engineering Mechanics Division, ASCE, Vol. 101, No. EM 6, Proc. Paper 11812, pp. 819-838, Dec. , 1975.

2) Abdel-Rohman, M. and Leipholz, H. H. : Structual Control by Pole Assignment Method, Journal of the Engineering Mechanics Division, pp. 1159-1175, Oct., 1978.

3) Yang, J-N, Akbarpour A. and Ghaemmaghami, P. : New Optimal Control Algorithms for Structual Control, Journal of Engineering Mechanics Division, ASCE, Vol.113, No.9, Proc. Paper 21800, pp. 1369-1386, Sep., 1987.

4) Rodellar, J.H., Barbat A. H. and Martin-Sanchez, J. M. : Predictive Control of Structures, Journal of the Engineering Mechanics Division, ASCE, Vol.113, No. 6 Proc. Paper 21538, pp. 797-812, June, 1987.

5) Fukazawa, K. and Kawahara, M. : Optimal Control of Structure Subjected to Earthquake Loads Using Dynamic Programing, Journal of Structual Engineering, JSCE, Vol. 34 A, pp. 759-766, March, 1988 (In Japanese).

6) Leipholz, H. H. E. and Abdel-Rohman, M. : Control of Structures, 1986, Martinus Nijhoff Publishers.

7) Meier, III, L. Larson, R. E. and Anthony, T. J. Tether. : Dynamic Programming for Stochastic Control Systems, IEEE, Vol. 16, No.6, pp. 767-775, December, 1971.

(Received October 7 1988) 